\title{
Análise sobre a fitoterapia como prática integrativa no Sistema Único de Saúde
}

\author{
SANTOS, R.L.*; GUIMARAES, G.P.; NOBRE, M.S.C.; PORTELA, A.S. \\ Departamento de Farmácia, Universidade Estadual da Paraíba, Avenida das Baraúnas, 351, Campus Universitário, \\ Bodocongó, CEP:58109-753, Campina Grande-Brasil *ravelylucena@hotmail.com
}

\begin{abstract}
RESUMO: É notável o crescente uso da fitoterapia como prática médica integrativa em diversos países. A utilização de plantas medicinais no Brasil tem como facilitadores a grande diversidade vegetal e o baixo custo associado à terapêutica, o que vem despertando a atenção dos programas de assistência à saúde e profissionais. O Ministério da Saúde, com a finalidade de evitar o uso inadequado desta prática medicinal, tem demonstrado interesse por meio do incentivo de pesquisas relacionadas ao assunto, favorecendo a implantação de programas de saúde visando à distribuição e utilização destes medicamentos de forma racional. Baseado neste contexto foi realizado levantamento de como esta temática vem sendo abordada e implementada no Sistema Único de Saúde (SUS). Tratase de estudo descritivo com abordagem qualitativa, realizado através de levantamento bibliográfico em bases de dados relacionado ao tema "medicamentos fitoterápicos no sistema único de saúde". Os descritores utilizados durante toda a pesquisa foram fitoterapia, medicamentos fitoterápicos, medicina integrativa, medicamentos, medicina herbária, fitoterapia no SUS, medicina integrativa e sistemas públicos de saúde. Observou-se que o governo tem demonstrado interesse no desenvolvimento de políticas que associem o avanço tecnológico ao conhecimento popular em prol de procedimentos assistenciais em saúde que apresentem eficácia, abrangência, humanização e menor dependência com relação à indústria farmacêutica. Nas duas últimas décadas, alguns estados e municípios brasileiros vêm realizando a implantação de Programas de Fitoterapia na atenção primária à saúde, com o intuito de suprir as carências medicamentosas de suas comunidades. Apesar da crescente busca por integrativas medicamentosas, os estudos acerca da fitoterapia ainda são precários no Brasil, fazendo-se ainda necessárias pesquisas nesta área, de modo a ampliar o conhecimento dos profissionais e estudantes da saúde, auxiliando e tornando mais sólidas as bases de segurança e eficácia para implementação das praticas fitoterápicas no SUS.
\end{abstract}

Palavras-chave: medicina integrativa, sistema único de saúde, fitoterapia

\begin{abstract}
Analysis about phytotherapy as an integrating practice in the Brazilian Unified Health System (UHS). The growing use of phytotherapy as an integrating medical practice in several countries has been remarkable. The use of medicinal plants in Brazil is facilitated by the plant diversity and low cost associated with therapeutics, which has called the attention of health assistance programs and professionals. The Brazilian Ministry of Health, in order to avoid misuse of this medical practice, has demonstrated interest, developing policies that encourage research related to this issue and favoring the establishment of health programs focused on the distribution and use of these drugs in a rational way. Based on this context, a survey about how this issue has been addressed and implemented in the Unified Health System (UHS) was carried out. This was a descriptive study of qualitative approach, performed through a literature review in databases, related to the theme "phytotherapic medicines in the unified health system". The key words used throughout the study were: phytotherapy, phytomedicines, integrating medicine, medicines, herbal medicine, phytotherapy in the UHS, integrating medicine and public health systems. The government has shown interest in developing policies which combine technological advances with common knowledge for the sake of assistance health procedures that show effectiveness, coverage, humanization and less dependence on the pharmaceutical industry. In the last two decades, some Brazilian states and municipalities have implemented Phytotherapy Programs in primary health care in order to meet the drug needs of their communities. Despite the growing search for integrating drugs, studies about phytotherapy are still limited in Brazil, with the need of research in this area in order to increase the knowledge of health professionals and students, helping and making more solid the safety and efficacy bases for the implementation of phytotherapic practices in the UHS.
\end{abstract}

Key words: integrating medicine, unified health system, phytotherapy

Recebido para publicação em 11/12/2009

Aceito para publicação em 07/07/2011

Rev. Bras. PI. Med., Botucatu, v.13, n.4, p.486-491, 2011. 
A Organização Mundial de Saúde (OMS) constatou que práticas não convencionais de saúde, tais como acupuntura, fitoterapia e técnicas manuais estão em desenvolvimento, ganhando espaço de modo complementar às terapias medicamentosas alopáticas (OMS, 2008).

Para grande parte da população o uso de plantas medicinais é visto como uma integrativa histórica à utilização de medicamentos sintéticos, visto que os últimos são considerados mais caros e agressivos ao organismo. A disseminação do uso de plantas medicinais, assim como a automedicação deve-se principalmente ao baixo custo e fácil acesso à grande parcela da população (OMS, 2008).

Visando o custo de desenvolvimento dessa categoria de produtos, os países subdesenvolvidos como o Brasil oferecem integrativa terapêutica bastante promissora para a população. O país é visto em destaque por possuir um terço da flora mundial, além de ser a Amazônia a maior reserva de produtos naturais com ação fitoterápica do planeta. Esta intensa presença vegetal faz com que as pesquisas e o próprio desenvolvimento de medicamentos fitoterápicos possam ocorrer como destaque no cenário científico mundial (Yunes et al., 2001; França et al., 2008).

Desde a década de 80 , vários documentos vêm sendo elaborados a fim de enfatizar o uso de fitoterápicos na atenção básica no sistema de saúde pública com o intuito de priorizar a melhoria dos serviços, o aumento da resolutividade e o incremento de diferentes abordagens (Matos, 1998).

O Ministério da Saúde através da Portaria no 971 de 3 de maio de 2006 disponibiliza opções terapêuticas e preventivas aos usuários do SUS, dentre elas o uso de plantas medicinais e medicamentos fitoterápicos alem de afirmar, baseado em levantamento realizado em 2004, que 116 municípios de 22 estados brasileiros fazem uso da fitoterapia (Brasil, 2006a).

Portanto, os tratamentos medicinais de origem vegetal são amplamente utilizados no Brasil como integrativa terapêutica, em destaque por aqueles que estão em tratamento de doenças crônicas e fazendo uso de outros medicamentos (Alexandre et al., 2008).

Embora existam vários estudos a respeito do uso, da toxicidade e da eficácia das plantas medicinais, a literatura científica ainda é precária no sentido de se conhecer como elas estão sendo usadas, quais são os benefícios e como se poderá capacitar os profissionais para o aconselhamento da utilização como medicina integrativa no SUS.

Diante desta abordagem, o objetivo desta pesquisa é fazer levantamento bibliográfico, a respeito dos temas como importância da fitoterapia; como ela está sendo usada; benefícios que a mesma oferece ao Sistema Público de Saúde; capacitação dos profissionais nesta área e programas e leis para a implementação no SUS.
Trata-se de estudo descritivo com abordagem qualitativa, realizado através de levantamento bibliográfico relacionado ao tema "A fitoterapia como medicina integrativa no Sistema Único de Saúde". Os descritores utilizados durante toda a pesquisa foram fitoterapia, medicamentos fitoterápicos, medicina integrativa, medicamentos, medicina herbária, fitoterapia no SUS, medicina integrativa e sistemas públicos de saúde e planta medicinal.

\section{Importância da fitoterapia}

A revitalização das práticas médicas antigas, hoje consideradas medicina integrativa, é um fenômeno que contribui para a forma hegemônica gradual destas modalidades, uma vez que sua organização mais ampla e integrada permite respostas mais apropriadas aos problemas gerados pela mecanicista especialização excessiva dos métodos médicos convencionais(Queiroz, 2000).

A fitoterapia vem sendo a medicina integrativa que mais cresce ao logo dos anos. No mercado mundial de medicamentos a comercialização de fitofármacos gira em torno de 15 bilhões de dólares. O fator mais relevante para tal crescimento se resume na evolução dos estudos científicos, em destaque a descoberta da eficácia de plantas medicinais, principalmente as utilizadas pela população com finalidade terapêutica, através dos estudos químicos e farmacológicos (Cechinel-Filho \& Yunes, 1998).

A população em geral confunde a fitoterapia com o uso de plantas medicinais. A Agência Nacional de Vigilância Sanitária (ANVISA) considera como medicamento fitoterápico aquele obtido exclusivamente de matérias-primas de origem vegetal, com qualidade constante e reprodutível e que tantos os riscos quanto à eficácia seja caracterizada por levantamentos etnofarmacológicos, documentações técnico científicas em publicações ou ensaios clínicos (Nicoletti et al., 2007).

As profundas raízes culturais da população brasileira facilitaram a sobrevida da Fitoterapia até os dias atuais, uma vez que a consciência popular reconhece a eficácia e legitimidade desta modalidade terapêutica (Sacramento, 2000). A crença popular em Curandeiros, raizeiros, parteiras, médiuns e a própria tradição oral ancestral transforma o uso de plantas medicinais em verdadeiro sincretismo de concepções (Santos et al., 1995).

Além disso, a fitoterapia faz com que o ser humano volte a se conectar com a natureza e assim buscar na vegetação uma forma de ajudar o organismo em vários sentidos, como a restaurar a imunidade enfraquecida, normalizar funções fisiológicas, desintoxicar órgãos e até mesmo para rejuvenescer (França et al., 2008).

\section{Como a fitoterapia está sendo usada}

Desde a antiguidade as pessoas utilizam 
plantas com a finalidade de tratar e curar enfermidade. Hoje podemos encontrar nas grandes e pequenas cidades brasileiras a comercialização de plantas medicinais livremente em feiras livres, mercados populares, quitandas residenciais e em outros estabelecimentos (Maciel et al., 2002). Esses produtos são comercializados por ervanários e raizeiros que não cumprem com os critérios de adequação à saúde nem atendem ao binômio segurança e eficácia exigidos nos demais produtos, mostrando assim uma comercialização inadequada e consequentemente má utilização das plantas medicinais, já que essas, neste caso, não passam por sistemas de qualificação da farmacovigilância (Brandão et al., 1998).

Rang et al. (2007) relatam que "A utilização de chás, de forma indiscriminada, em crianças portadoras de enfermidades hepáticas, renais ou outras doenças, poderá Ihes trazer sérias conseqüências para sua saúde se não houver acompanhamento médico". Wong (2003) afirma que a utilização indiscriminada de remédios e associação de fármacos aumenta em toda a população o risco de morbimortalidade causados pelos efeitos adversos e toxicidade provocada por estes produtos.

Antes de serem utilizadas pela população é necessário que as plantas medicinais passem por vários processos que no final vão chegar a formulações com indicações de uso seguro e adequado para assim fornecer resultados desejados a quem for utilizá-la. Tais processos englobam a química orgânica, fitoquímica (onde ocorre o isolamento, purificação e caracterização de princípio ativo), a farmacologia (investigando farmacologicamente os extratos e os constituintes químicos isolados), a química orgânica sintética (fazendo as transformações químicas de princípios ativos) a química medicinal e farmacológica (estudando a relação estrutura/atividade e os mecanismos de ação dos princípios ativos) e por fim a preparação de formulações para a produção do fitoterápico (Maciel et al., 2002).

\section{Os benefícios da fitoterapia para o Sistema Público de Saúde}

No ocidente, considera-se a Alemanha como primeiro e maior incentivador das terapias naturais, notadamente a Fitoterapia, uma vez que no receituário alemão os produtos florais chegam a ocupar cerca de $40 \%$ das prescrições. Há também países como a França, Bélgica, Suécia, Suíça, Japão e Estados Unidos onde se enfatiza a técnica fitoterápica e onde os trabalhos científicos sobre o tema são publicados. A China é campeã na utilização de medicamentos naturais. Naquele País só se recorre à alopatia quando não se encontra um substituto de tal medicamento na flora chinesa (Leão \& Ribeiro, 1999).

Visando a eficácia e o baixo custo operacional da utilização de plantas medicinais nos programas de atenção primária à saúde, pode-se considerar uma integrativa terapêutica muito útil e importante. A facilidade para adquirir essas plantas e a compatibilidade cultural são fatores de extrema relevância para o progresso dessa medicina, principalmente no Nordeste brasileiro onde na cultura é comum o uso das mesmas na preparação de remédios caseiros para tratar varias enfermidades. Além disso, o fato de plantas medicinais poderem ser usadas através de formulações caseiras, de fácil preparo, se reveste de grande importância, pois ela pode suprir a crônica falta de medicamentos nos serviços de saúde (Matos, 1998).

Essa idéia vem sendo desenvolvida já algum tempo. Segundo Boas e Gadelha (2007), o relatório final da 10a Conferência Nacional de Saúde, em 1998, determina que os gestores do SUS devam estimular e ampliar pesquisas realizadas em parceria com universidades públicas, promovendo ao lado de outras terapias complementares a fitoterapia.

No Brasil, diretrizes do Ministério da Saúde determinaram prioridades na investigação das plantas medicinais e implantando a fitoterapia como prática oficial da medicina, orientando as Comissões Interinstitucionais de Saúde (CIS) a buscarem sua inclusão no SUS. Para que essa inclusão ocorra é essencial que os profissionais da área de saúde conheçam as atividades farmacológicas e a toxicidade das plantas medicinais de cada bioma brasileiro, de acordo com os costumes, tradições e condição sócio-econômica da população. Alguns trabalhos já são realizados em estados como o Ceará com o objetivo de desvendar o uso de plantas medicinais pela população, encontrando alta prevalência de uso (Silva et al., 2006).

\section{Como capacitar profissionais para a fitoterapia}

Embora a área de fitoterápicos esteja cada vez mais conhecida e desenvolvida, a disponibilidade de produtos fitoterápicos no mercado brasileiro ainda é bastante preocupante na visão do controle de qualidade desde a produção até a comercialização e uso pela população, pois a capacitação de profissionais nesta área ainda é escassa (Calixto, 2000).

Guiados pela mídia, as pessoas utilizam os produtos provenientes do reino vegetal sob a forma de complementos e suplementos alimentares, sem levar em consideração o potencial curativo destes medicamentos. Outros fatores que influenciam na préconcepção mistificadora ocidental é a associação direta dos fármacos fitoterápicos com práticas integrativas de medicina, como a Medicina Chinesa, a Medicina Holística, Macrobiótica entre outras (Camargo, 1985).

A fitoterapia é considerada pela OMS uma

Rev. Bras. PI. Med., Botucatu, v.13, n.4, p.486-491, 2011. 
prática da medicina tradicional (Simon, 2001). Todavia, o uso deve ser precedido por criteriosa identificação e classificação botânica a fim de evitar a indução de erros e problemas durante a utilização. Muitas vezes os profissionais de saúde associam o uso destes medicamentos com os medicamentos alopáticos (França et al., 2008), o que pode gerar riscos, uma vez que diversas interações têm sido descritas entre fitoterápicos e fármacos quimicamente definidos, algumas associadas à modulação da atividade enzimática no sítio de atuação (Franco, 2003).

Há preocupação ainda maior quanto aos herbolários (pessoas que comercializam plantas medicinais), pois estes obtêm os conhecimentos geralmente advindos da tradição oral, sem o adequado respaldo científico. Muitos deles não estão preparados para avaliar critérios importantes no tratamento medicamentoso como o armazenamento, uso, indicações e contra-indicações das plantas medicinais. Em estudo realizado na cidade de Campina Grande - Paraíba foi demonstrado que poucos herbolários associam o conhecimento adquirido com a tradição oral à literatura acerca do tema (França et al., 2008).

Os profissionais de saúde devem levar em conta que uma gama de fatores interfere na atuação do complexo ativo obtido diretamente dos vegetais. Aspectos genéticos dos indivíduos podem expressar diferentes atividades das substâncias bioativas presentes, e estes podem ser afetados também por condições climáticas como luminosidade, índice pluviométrico, condições do solo e outros fatores. Faz-se necessário conhecer mais acerca da taxonomia do material botânico a ser utilizado, uma vez que os fitoterápicos apresentam altos risco de efeitos colaterais e intoxicações (França et al., 2008).

Segundo a Política Nacional de Práticas Integrativas e Complementares (PNPIC) no SUS, a capacitação, na área de "Plantas medicinais e Fitoterapia", deve ser realizada através de curso básico interdisciplinar comum a toda a equipe, visando à sensibilização dos profissionais a respeito dos princípios e diretrizes do SUS, das políticas de saúde, das Práticas Integrativas no SUS, das normas e regulamentação e dos cuidados gerais com as plantas medicinais e fitoterápicos; cursos específicos para profissionais de saúde de nível universitário, detalhando os aspectos relacionados à manipulação, fitoterápicos, de acordo com as categorias profissionais e cursos específicos para profissionais da área agronômica detalhando os aspectos relacionados a toda cadeia produtiva de plantas medicinais (Brasil, 2006a).

A fim de seguir as recomendações da PNPIC, o Ministério da Saúde, responsável pela Política de Educação na Saúde, conta com estratégias para formação e educação dos profissionais de saúde, como o Sistema Universidade Aberta do SUS (UNA-SUS); 0
Programa Nacional de Telessaúde; o Programa de Educação Permanente pelo Trabalho para a Saúde; Cursos de Especialização e Mestrado Profissionalizante; entre outros. Alem de contar com essas estratégias o Ministério da Saúde tem incentivado a inclusão de disciplinas de interesse do SUS nos currículos dos cursos de graduação na área da saúde e inserido disciplinas sobre fitoterapia e outras práticas integrativas e complementares nos cursos de especialização que por eles são financiados (Simoni, 2010).

\section{Medidas para implantação da fitoterapia no SUS}

Com o crescimento da utilização e desenvolvimento de medicamentos fitoterápicos, a OMS tem demonstrado crescente interesse em melhorar as condições que garantam qualidade, segurança e eficácia destes produtos, focando especialmente o seu uso em países em vias de desenvolvimento (BRASIL, 2000). Com o objetivo de evitar efeitos irracionais e danos que possam ser causados pelo mau uso desta modalidade terapêutica, - Ministério da Saúde tem estabelecido políticas que encorajam o desenvolvimento de estudos com plantas medicinais objetivando colocar em prática os benefícios advindos destas pesquisas (Franco, 2003).

O governo tem mostrado interesse no desenvolvimento de políticas e programas que associem o conhecimento popular com o científico, assim ao longo de vários anos vem sendo criado portarias e programas relacionada a plantas medicinais e fitoterápicos no SUS. Dentre estes merecem destaques:

- O Programa de Pesquisa de Plantas Medicinais (PPPM) da Central de Medicamentos (CEME), criado pelo ministério da saúde e vigente durante 15 anos (1982 e 1997), teve como objetivo principal estudar 55 plantas medicinais com o intuito de determinar a ação terapêutica, que estas plantas tinham segundo a população, a partir de estudos científicos e assim desenvolver uma terapia alternativa e complementar (Simoni, 2010). Cinco anos após a PPPM iniciar, foi criado a Resolução da Comissão Interministerial de Planejamento e Coordenação (CIPLAN) no 08, em 08 de março de 1988, que determinava procedimentos e rotinas relativas à prática da Fitoterapia nas Unidades Assistenciais Médicas e consequentemente regulamentava a prática de Fitoterapia nos serviços de saúde (Brasil, 1988).

- No ano de 1998 foi aprovado a Política Nacional de Medicamentos, Portaria № 3916, que estabelecia a expansão do apoio às pesquisas destinadas a fitoterápicos, visando o potencial terapêutico da flora e fauna nacionais, uma vez que - Brasil é o país de maior biodiversidade do planeta (Brasil, 1998). Em meados de em 2001, o Ministério da Saúde realizou um Fórum para formulação da 
proposta da Política Nacional de Plantas Medicinais e Medicamentos Fitoterápicos, porém esta só foi aprovada no ano de 2006 pelo decreto Presidencial o 5.813, de 22 de junho de 2006 (Brasil, 2006b). A fim de definir e pactuar as ações que visam o uso de plantas medicinais e fitoterápicos no processo de atenção à saúde, respeitando desde o conhecimento tradicional até o uso da biodiversidade do país, o Conselho Nacional de Saúde em 2004 aprovou a Política Nacional de Assistência Farmacêutica com a resolução no 338 .

- 1-Conferência Nacional de Medicamentose Assistência Farmacêutica no ano de 2005 aprovou 48 recomendações, e entre elas a implantação de programas para uso de medicamentos fitoterápicos nos serviços de saúde, diante disto em 2006, através da portaria MS no 971, foi aprovada a Política Nacional de Práticas Integrativas e Complementares (PNPIC) no SUS, que trata das diretrizes, ações e responsabilidades dos três governos, federal, estadual e municipal, os quais devem ofertar nos serviços de saúde: serviços e produtos homeopáticos, plantas medicinais e fitoterápicos, medicina tradicional chinesa/acupuntura, entre outros. Deve ficar claro que a essa política tem o objetivo de ampliar o acesso a opções de tratamentos com produtos e serviços seguros, eficazes e de qualidade, de forma integrativa e complementar e não em substituição ao modelo convencional, por tanto estes programas devem dispor de profissionais devidamente capacitados, e os produtos devem atender obrigatoriamente os critérios de segurança, qualidade e eficácia terapêutica (Simoni, 2010).

- A Política Nacional de Plantas Medicinais e Fitoterápicos, aprovada pelo Decreto Presidencial № 5.813, de 22/06/2006, foi elaborada por Grupo de Trabalho Interministerial (GTI) o qual contempla diretrizes que vão desde a cadeia produtiva de plantas medicinais ate os produtos fitoterápicos (Brasil, 2006b). Essa política fez surgir um novo GTI para elaborar o Programa Nacional de Plantas Medicinais e Fitoterápicos cuja proposta foi submetida à consulta pública e aprovado em 09/12/2008, por meio da Portaria Interministerial № 2.960, que além de criar essa GTI, criou o Comitê Nacional de Plantas Medicinais e Fitoterápicos com a missão de monitorar e avaliar a implantação da Política Nacional.

- Com a finalidade de instituir o programa Farmácia Viva no Sistema Único de Saúde (SUS) em 2010 foi aprovada a Portaria no 886/GM/MS. O programa Farmácia Viva foi criado pelo professor Francisco José de Abreu Matos da Universidade Federal do Ceará, que estudou por mais de 50 anos plantas medicinais e originou vasta e reconhecida literatura científica sobre estas plantas e uso. $O$ programa foi o primeiro de assistência farmacêutica baseado no emprego científico de plantas medicinais desenvolvido no Brasil, tendo por objetivo produzir medicamentos fitoterápicos acessíveis à população (Matos, 1998) e realizar todas as etapas do cultivo, coleta, processamento, armazenamento de plantas medicinais, manipulação, dispensação de preparações magistrais até oficinas de plantas medicinais e produtos fitoterápicos (Simoni, 2010).

Nas duas últimas décadas, alguns estados e municípios brasileiros vêm realizando a implantação desses programas e portarias na atenção primária à saúde, com o intuito de suprir as carências medicamentosas de comunidades, e muitos desses programas estão, atualmente, vinculados ao programa saúde da família (Ogava et al., 2003; Michilis, 2004).

\section{Considerações finais}

Diante da biodiversidade do Brasil e do objetivo de melhorar a saúde da população, o Ministério da Saúde vem investindo no uso da fitoterapia como complemento para o SUS. Entretanto, para que isso ocorra de forma correta e, principalmente, segura é necessário profissionais capacitados, que compreendam a química, toxicologia e farmacologia das plantas medicinais e princípios ativos sem desconsiderar o conhecimento popular.

Existem diversos programas de fitoterapia implantados ou em fase de implantação, em todas as regiões do Brasil. Isto se deve a busca das Secretarias Municipais de Saúde em facilitar o acesso da população às plantas medicinais/fitoterápicos visando o uso correto das mesmas. Está pratica está sendo, à priori, implantada no Programa Saúde da Família (PSF) de diversos estados.

Apesar da crescente busca por integrativas medicamentosas, os estudos acerca da fitoterapia ainda são precários no Brasil, sendo necessário o desenvolvimento de pesquisas nesta área, que enriqueceriam o conhecimento dos profissionais e estudantes da saúde, auxiliando e tornando mais seguras e eficazes a implementação das práticas fitoterápicas no SUS.

\section{REFERÊNCIA}

ALEXANDRE, R.F.; BAGATINI, F.; SIMÕES, C.M.O. Interações entre fármacos e medicamentos fitoterápicos à base de ginkgo ou ginseng. Revista Brasileira de Farmacognosia, v.18, n.1, p.117-26, 2008.

BOAS, G.K.V.; GADELHA, C.A.G. Oportunidades na indústria de medicamentos e a lógica do desenvolvimento local baseado nos biomas brasileiros: bases para a discussão de uma política nacional. Caderno de Saúde Pública, v.23, n.6, p.1463-71, 2007.

BRANDÃO, M.G.L.; FREIRE, N.; VIANNA-SOARES, C.D. Vigilância de fitoterápicos em Minas Gerais. Verificação da qualidade de diferentes amostras comerciais de camomila. Caderno de Saúde Pública, v.14, n.3, p.61316, 1998. 
BRASIL. Comissão Interministerial de Planejamento e Coordenação - CIPLAN. Resolução № 5 de 08 de março de 1988. Brasília, 1988.

BRASIL. Ministério da Saúde. Agência Nacional de Vigilância Sanitária. Resolução RDC 17 de 24 de fevereiro de 2000. Aprova o Regulamento técnico visando normatizar o registro de medicamentos fitoterápicos junto ao sistema de vigilância sanitária. Diário Oficial da União, Brasília, DF, 25 Fev. 2000.

BRASIL. Ministério da saúde. Portaria no 971 de 3 de maio de 2006. Brasília, 2006a.

BRASIL. Decreto Presidencial no 5813, de 22 de junho de 2006. Política Nacional de Práticas Integrativas e Complementares no SUS. Brasília, 2006b.

BRASIL. Ministério da Saúde. Portaria MS/GM no 3916, de 30 de outubro de 1998. Brasília, 1998.

CALIXTO, J.B. Efficacy, safety, quality control, marketing and regulatory guidelines for herbal medicines (phytotherapeutic agents). Brazilian Journal of Medical and Biological Research, v.33, n.2, p.179-89, 2000.

CAMARGO, M.T.L.A. Medicina popular: aspectos metodológicos para pesquisa, garrafada, objeto de pesquisa, componentes medicinais de origem vegetal, animal e mineral. São Paulo: ALMED, 1985. 130p.

CECHINEL-FILHO, V.; YUNES, R.A. Estratégias para a obtenção de compostos farmacologicamente ativos a partir de plantas medicinais. Conceitos sobre modificação estrutural para otimização da atividade. Química Nova, v.21, n.1, p.99-105, 1998.

FRANÇA, I.S.X. et al. Medicina popular: benefícios e malefícios das plantas medicinais. Revista Brasileira de Enfermagem, v.61, n.2, p. 201-8, 2008.

FRANCO, L.L. Doenças tratadas com plantas medicinais. 2.ed. Rio de Janeiro: Vozes, 2003.

LEÃO, M.G.; RIBEIRO, K.L.M.S. Subprojeto de Plantas Medicinais. Proposta Preliminar do Plano Municipal de Desenvolvimento Rural-PP/PMDR. Comodoro: Conselho Municipal de Desenvolvimento Rural-CMDR Prefeitura Municipal de Comodoro, Estado de Mato Grosso-Brasil, 1999. 45p.

MACIEL, M.A.M. et al. Plantas medicinais: a necessidade de estudos multidisciplinares. Química Nova, v.25, n.3, p.429-38, 2002.

MATOS, F.J.A. Farmácias vivas: sistema de utilização de plantas medicinais projetado para pequenas comunidades. 3.ed. Fortaleza: EUFC, 1998. 219p.
MICHILIS, E. Diagnóstico situacional dos serviços de fitoterapia no Estado do Rio de Janeiro. Revista Brasileira de Farmacognosia, v.14, supl.1, p.16-9, 2004. NICOLETTI, M.A. et al. Principais interações no uso de medicamentos fitoterápicos. Infarma, v.19, n.1, p.32-50, 2007.

OGAVA, S.E.M. et al. Implantação do programa de fitoterapia "Verde Vida" na secretaria de saúde de Maringá (2000-2003). Revista Brasileira de Farmacognosia, v.13, supl.1, p.58-62, 2003.

ORGANIZAÇÃO MUNDIAL DE SAÚDE. Traditional medicine: definitions. Disponível em: <http://www.who. int/medicines/areas/traditional/definitions/en/>. Acessado em: 10 de set. 2008.

QUEIROZ, M.S. O itinerário rumo às medicinas integrativas: uma análise em representações sociais de profissionais da saúde. Caderno de Saúde Pública, v.16, p.363-75, 2000

RANG, H.P.; DALE, M.M.; RITTER, J.M. Farmacologia. 6.ed. Rio de Janeiro: Elsevier, 2007. 848p.

SACRAMENTO, H.T. Legislação para produção, comercialização e uso de plantas medicinais. In: JORNADA PAULISTA DE PLANTAS MEDICINAIS, 5; 2001. Botucatu. Anais... Botucatu: UNESP, 2000. p.33. SANTOS, M.G.; DIAS; A.G.P.; MARTINS, M.M. Conhecimento e uso da medicina integrativa entre alunos e professores de primeiro grau. Revista Saúde Pública, v.29, n.3, p.221-7, 1995.

SILVA, M.I.G. et al. Utilização de fitoterápicos nas unidades básicas de atenção à saúde da família no município de Maracanaú (CE). Revista Brasileira de Farmacognosia, v.16, n.1, p.455-62, 2006.

SIMON, D.R.; CHOPRA, D. O guia Deepak Chopra de ervas: 40 receitas naturais para uma saúde perfeita. 1.ed. Rio de Janeiro: Campus, 2001. 272p.

SIMONI, C.L. Fitoterapia - linha do tempo. Disponível em: <http://portal.saude.gov.br/ profissional>. Acesso em: 10 de out. 2010.

WONG, A. Os usos inadequados e os efeitos adversos de medicamentos na prática clínica. Jornal de Pediatria, v.79, n.5, p.378-80, 2003.

YUNES, R.A.; PEDROSA, R.C.; CECHINEL-FILHO, V. Fármacos e fitoterápicos: a necessidade do desenvolvimento da indústria de fitoterápicos e fitofármacos no Brasil. Química Nova, v.24, n.1, p.14752, 2001. 